\title{
Do island populations have less genetic variation than mainland populations?
}

\author{
R. FRANKHAM* \\ Key Centre for Biodiversity and Bioresources, Macquarie University, Sydney, NSW 2109, Australia
}

\begin{abstract}
Island populations are much more prone to extinction than mainland populations. The reasons for this remain controversial. If inbreeding and loss of genetic variation are involved, then genetic variation must be lower on average in island than mainland populations. Published data on levels of genetic variation for allozymes, nuclear DNA markers, mitochondrial DNA, inversions and quantitative characters in island and mainland populations were analysed. A large and highly significant majority of island populations have less allozyme genetic variation than their mainland counterparts (165 of 202 comparisons), the average reduction being 29 per cent. The magnitude of differences was related to dispersal ability. There were related differences for all the other measures. Island endemic species showed lower genetic variation than related mainland species in 34 of 38 cases. The proportionate reduction in genetic variation was significantly greater in island endemic than in nonendemic island populations in mammals and birds, but not in insects. Genetic factors cannot be discounted as a cause of higher extinction rates of island than mainland populations.
\end{abstract}

Keywords: allozymes, conservation, endemic species, extinction, genetic variation, islands.

\section{Introduction}

Island populations have a much higher risk of extinction than mainland populations (Diamond, 1984; Vitousek, 1988; Flesness, 1989; Case et al., 1992; World Conservation Monitoring Centre, 1992; Smith et al., 1993). Recorded extinctions since 1600 show that a majority of extinctions of mammals, birds and reptiles were of insular forms, and substantial proportions of extinctions in invertebrates and vascular plants were of island forms (Table 1), even though island species represent a minority of total species in all groups. For example, only 20 per cent of all bird species are on islands, but 90 per cent of bird species driven to extinction in historic times have been island dwellers (Myers, 1979). Further, substantial proportions of endangered and vulnerable species are of insular species (Table 2). Endemic species are particularly prone to extinction or endangerment. Davis et al. (1986) reported 55-97 per cent of endemic plant taxa on 13 islands were extinct, endangered, rare or threatened, and Reid \& Miller (1989) described 6-94 per cent

\footnotetext{
'Islands are the key' (E. O. Wilson, 1994). *E-mail: rfrankha@rna.bio.mq.edu.ac
}

of endemic plant species as threatened on 15 islands.

Human activities have been the major cause of species extinctions on islands in the past 50000 years (Olson, 1989) through over-exploitation, habitat loss and introduced species. The relative importance of these factors in recorded extinctions varies according to the taxonomic group. Reid \& Miller (1989) rated over-exploitation and introduced species as the most important causes in vertebrates, followed by habitat loss, whereas the World Conservation Monitoring Centre (1992) reported introduced animals and habitat loss to be the most important cause of animal extinctions, followed by over-exploitation. Both report substantial proportions of cases where the cause was unknown. Habitat loss is the most important cause of endangerment in all vertebrate taxa, except for reptiles where it is second to overexploitation (Reid \& Miller, 1989). The role of disease is not listed in these compilations. However, there is a growing suspicion that new and changed diseases represent a significant factor (Diamond, 1984; Dobson \& May, 1986; O’Brien \& Evermann, 1988; Flesness, 1989).

The reason for the susceptibility of island populations to extinction is controversial. The coup de grâce is usually delivered by stochastic factors, whether 
Table 1 Recorded extinctions, 1600 to present

\begin{tabular}{lrrrrc}
\hline Taxa & Island & Mainland & Ocean & Total & Per cent island \\
\hline Mammals* & 34 & 24 & - & 58 & 59 \\
Birds* & 104 & 11 & - & 115 & 90 \\
Reptiles $\dagger$ & 20 & 1 & 0 & 21 & 95 \\
Amphibians $\dagger$ & 0 & 2 & 0 & 2 & 0 \\
Fish $\dagger$ & 1 & 22 & 0 & 23 & 4 \\
Molluscs* & 151 & 40 & - & 191 & 79 \\
Invertebrates $\dagger$ & 48 & 49 & $\mathbf{1}$ & 98 & 49 \\
Vascular plants $\dagger$ & 139 & 245 & 0 & 384 & 36 \\
\hline
\end{tabular}

*From World Conservation Monitoring Centre (1992).

†From Reid \& Miller (1989).

Table 2 Endangered and vulnerable species (from Reid \& Miller, 1989)

\begin{tabular}{lrrrrc}
\hline Taxa & Island & Mainland & Ocean & Total & Per cent island \\
\hline Mammals & 48 & 159 & 9 & 216 & 22 \\
Birds & 87 & 91 & 0 & 178 & 49 \\
Reptiles & 21 & 41 & 6 & 68 & 31 \\
Amphibians & 0 & 14 & - & 14 & 0 \\
Fish & 21 & 443 & 0 & 464 & 5 \\
Invertebrates & 338 & 371 & 2 & 711 & 48 \\
Vascular plants & 2706 & 3985 & 0 & 6691 & 40 \\
\hline
\end{tabular}

demographic, environmental, catastrophic, or genetic. Pimm (1991) and other ecologists stress the susceptibility of small island populations to demographic and environmental stochasticity. However, the susceptibility of island populations is predicted on genetic grounds (Frankham, 1995a). Oceanic island populations are expected to lose genetic variation at foundation (as few as a single individual in self-fertilizing species, or a pair in nonselfing species). Both oceanic and land-bridge islands should lose genetic variation after foundation as they typically have lower population sizes than mainland populations.

Genetic variation is the raw material for evolutionary change (Frankel \& Soulé, 1981). Genetic variation allows populations to evolve in response to environmental change, whether that be new/changed diseases, parasites, predators and competitors, or greenhouse warming, ozone layer depletions, or other results of pollution. Pre-existing genetic variation is critical for short-term evolutionary change (Ayala, 1965; R. Frankham, E. Lowe, M. E. Montgomery, L. M. Woodworth, \& D. A. Briscoe, unpublished data) as the waiting times for new favourable mutations are high unless population sizes are very large. The IUCN (World Conservation
Union) has recognized genetic diversity as one of three levels of biological diversity requiring conservation (McNeely et al., 1990).

Genetic variation on islands is determined by the net effects of loss at foundation, subsequent loss caused by finite population size since foundation, and gains arising from secondary immigration and new mutations (Jaenike, 1973). Natural selection influences the loss of genetic variation; selection for a favourable allele will increase rate of loss, whereas heterozygote advantage may slow it.

A single population size bottleneck is predicted to reduce heterozygosity and evolutionary potential by $1 / 2 N_{\mathrm{e}}$ where $N_{\mathrm{e}}$ is the effective size of the bottleneck generation (James, 1971). For example, if an oceanic island population is founded from a single pair, heterozygosity is expected to be immediately reduced by 25 per cent. Populations that have been subjected to bottlenecks usually have reduced allozyme variation (see Leberg, 1992; Hartl \& Pucek, 1994) and quantitative genetic variation (Robertson, 1966; James, 1971; Frankham, 1980; Franklin, 1980; Brakefield \& Saccheri, 1994). Conversely, Bryant et al. (1986) and López-Fanjul \& Villaverde (1989) reported higher quantitative genetic variation for characters related to fitness in bottlenecked popula- 
tions. However, bottlenecked populations show reduced evolutionary potential (R. Frankham, P. R. England, K. E. L. Lees \& D. A. Briscoe, unpublished data).

Heterozygosity for neutral alleles is expected to show an approximately exponential decay with generations in finite populations, as described by eqn 1

$H_{\mathrm{t}} / H_{0}=\left[1-1 / 2 N_{\mathrm{e}}\right]^{t} \sim e^{-t / 2 N_{\mathrm{e}}}$.

where $H_{t}$ is the heterozygosity at time $t, H_{0}$ the original heterozygosity, $N_{\mathrm{e}}$ the effective population size, and $t$ the number of generations (Crow \& Kimura, 1970). A feature of this relationship is that the variance in heterozygosity is expected to increase in small populations, such that the relationship can be obscured unless there are many genetic markers and many replicates.

A correlation between heterozygosity and effective population size is also expected for loci under heterozygote advantage selection in finite populations. The effect of heterozygote advantage on fixation probability depends on the equilibrium frequency of the alleles (Robertson, 1962). Selection retards fixation for alleles with equilibrium frequencies in the 0.2-0.8 range. Conversely, selection accelerates fixation for alleles with equilibrium frequencies outside this range. Hence, heterozygote advantage in finite populations will slow fixation for some alleles and accelerate it for others. Alleles subject to natural selection approach effective neutrality as the effective population size drops, i.e. when the selection coefficient drops below $1 / 2 N_{\mathrm{e}}$ (Wright, 1931; Kimura, 1983). Selection on individual alleles detected by electrophoresis or DNA sequence is generally weak, so they will be subject to genetic drift unless population sizes are very large (Robertson, 1962; Kimura, 1983; Ohta, 1992; Satta et al., 1994).

The predicted relationship between genetic variation and effective population size given in eqn 1 has been verified for allozyme genetic variation in experimental populations of Drosophila (M. E. Montgomery, R. K. Nurthen, L. M. Woodworth, D. A. Briscoe \& R. Frankham, in preparation). Further, there are clear associations between population size and genetic variation in wildlife, both within and among species (reviewed by Frankham, 1996). For example, Soulé (1976) observed a correlation of 0.70 between allozyme heterozygosity and the logarithm of population size for animal species, explaining $\approx 1 / 2$ of the variation in heterozygosity.

Differences between island populations and main- land ones are expected to be greater when the number of founders is smallest, when the population size differences are greatest, and when the immigration rates are lowest. Differences should be greater for small rather than large islands, for species with lower dispersal rates, and for distant rather than near islands (Jaenike, 1973). Reversal of the difference is expected when mainland populations are derived from island populations (e.g. after glaciation), and where island populations are larger than mainland populations.

Endemic island species have no mainland counterparts, yet they represent the oldest and most differentiated island populations. According to eqn 1, they would be expected to have proportionately lower genetic variation than island species with mainland representatives as their time since foundation from a mainland population will typically be greater than for nonendemic island populations.

Surprisingly, the evidence on genetic variation in island populations is equivocal. Selander \& Johnson (1973) and Selander (1976) indicated there were differences, whereas Nevo (1978) reported that island populations of vertebrates had lower levels of genetic variation than mainland populations, but invertebrates did not show a significant difference. Conversely, the most recent comprehensive review by Nevo et al. (1984) found no significant difference for either group, though there was a trend in the predicted direction. Kilpatrick (1981) and Berry (1986) concluded that there were differences in mammals, while Boag (1988) concluded that island and mainland bird species do not differ. All these compilations were based on a small number of studies. Given the importance of the issue to the understanding of the susceptibility of island populations to extinction, it is critical that this issue be resolved.

The hypothesis that genetic factors contribute to the higher extinction rate of island populations predicts that island populations of sexually reproducing species will have, on average, lower levels of genetic variation than comparable mainland populations. Further, endemic island populations should have lower genetic variation than nonendemic island populations. The objective of this study was to test these hypotheses by reviewing and analysing published evidence.

\section{Collection and analyses of data}

Data were obtained from a literature survey of publications where comparisons were made of genetic variation in mainland and island populations 
of the same species, usually with the same loci surveyed. Literature was surveyed by checking all relevant previous reviews and the papers they referred to, by performing a keyword search of Biological Abstracts on CD ROM 1992-95, and by checking references that those papers made to other relevant studies. Data from all relevant studies were analysed using sign tests (see below). Marine species were excluded as island populations belong to the same continuous habitat over which they often disperse widely (Stepien \& Rosenblatt, 1991; Fevolden, 1992). All data were on independent populations, apart from those for Peromyscus polionotus where the same two island populations were studied by Selander et al. (1971), Garten (1976) and Brewer et al. (1990). The mean of the three studies was used in the sign tests (heterozygosities of 3.33 and 6.97 per cent for island populations vs. 6.98 per cent for mainland). The data of Bock \& McCracken (1988) on island populations of green iguanas from Gatun Lake were not included as the island populations were not isolated from the surrounding mainland population; animals from nearby mainland populations nest annually on the islands. The data of Wendel et al. (1992) on island vs. mainland populations of cotton (Gossypium hirsutum) were not analysed as its distribution is believed to be strongly influenced by human colonization and trade. Johnson \& Selander (1971) reported an overall figure of 2.3 per cent heterozygosity for two island populations of Dipodomys compactus, with no individual details of sample sizes. The Mustang Island population was monomorphic. One of two populations on Padre Island was monomorphic, whereas the other Padre Island population was polymorphic for one of 18 loci, with two alleles at frequencies of 0.33 and 0.67 (heterozygosity $=2.46$ per cent). A heterozygosity of 1.23 per cent was attributed to Padre Island (mean of 0.00 and 2.46 per cent). Only pooled data for several islands were reported for Drosophila nebulosa and D. tropicalis (Powell, 1975) so these were each treated as one island for the purposes of sign tests.

The null hypothesis is that island populations do not differ from mainland populations or have more variation, and the alternative hypothesis is that island populations have less genetic variation than comparable mainland populations. Consequently, one-tailed $\chi^{2}$ sign tests were used. Statistical analyses were performed on average heterozygosity values. Preference was given to using gene diversity (expected Hardy-Weinberg heterozygosity) as this is least affected by sample size. Where this was not available observed heterozygosity was used in analy- ses. Data where both the mainland and the island populations had no genetic variation are included in Table 3 , but were not used in the sign test as they are not informative.

Two sign tests were performed on the allozyme data for nonendemic populations, one where every island population was compared against the mean of its corresponding mainland populations, and a second where the mean of islands was compared with the mean of mainland populations for the same study. The latter test covers the possibility that there was migration to one island, and the other island populations were derived from that population. A Kruskal-Wallis test was used to determine whether taxa differed in the ratio of island to mainland heterozygosities. As ratios are not normally distributed, nonparametric statistics were used to compare them throughout this study. Statistical analyses were performed using the MINITAB statistical package release 7 . The ratios of heterozygosities for island: mainland populations of the different taxa were obtained by calculating mean heterozygosities for islands and mainland populations of each taxa and then computing the ratio of these.

Similar comparisons were made of genetic variation in endemic island species and the most related mainland species or group that was available. The data of Johnson et al. (1989) on Hawaiian honeycreepers were based on very small sample sizes for both island and mainland species, though 36 loci were typed. Further, there was uncertainty regarding the appropriate mainland species to use for comparisons. Taxonomic data presented in the paper indicated that the family Emberizidae was the appropriate mainland group, so this was used. Analyses were also performed using the mean of the families Emberizidae and Fringillidae as the mainland group. Only pooled data for the two island populations of the endemic Peromyscus sejugis were reported, so these data have been treated as a single point for sign tests. Avantazi et al. (1994) describe two endemic species of mites on the Canary Islands, but only one is reported here as their taxonomic separation is doubtful; they shared the same alleles at 14 allozyme loci and frequencies were similar at the one polymorphic locus. For the endemic plants in the genus Crepidiastrum, the 'mainland' species for comparison come from the much larger main Japanese islands (Ito \& Ono, 1990).

Comparisons of the ratio of heterozygosity in island:mainland populations were carried out for endemic vs. nonendemic species for the full data set, and for different taxa using Mann-Whitney nonparametric tests. 
Table 3 Allozyme genetic variation in island (Is) and mainland (M) populations, characterized as gene diversity $\left(H_{\mathrm{e}}\right.$ percentage), observed heterozygosity $\left(H_{\mathrm{o}}\right.$ percentage), allelic diversity $(A)$ and percentage of loci polymorphic $(P)$

\begin{tabular}{|c|c|c|c|c|c|c|c|}
\hline Species & Is vs. $\mathrm{M}$ & $H_{\mathrm{e}}$ & $H_{\mathrm{o}}$ & $A$ & $P$ & $\begin{array}{l}M>\text { Is: } \\
\text { M }<\text { Is* }\end{array}$ & Reference \\
\hline Mammals & & & & & & $82: 20$ & \\
\hline \multirow[t]{2}{*}{ Alces alces } & Is† & & 2.6 & & 8.6 & $1: 1$ & Ryman et al. (1980) \\
\hline & M & & 2.0 & & 9.8 & & \\
\hline \multirow[t]{2}{*}{ Canis lupus } & Is & 3.9 & 4.0 & 1.08 & 8.0 & $1: 0$ & Wayne et al. (1991b) \\
\hline & $\mathbf{M}$ & 8.7 & 6.1 & 1.20 & 20.0 & & \\
\hline \multirow[t]{2}{*}{ Cervus elaphurus atlanticus } & Is & & 0.0 & & 0.0 & - & Gyllensten et al. (1983) \\
\hline & $\mathbf{M}$ & & 0.0 & & 0.0 & & \\
\hline \multirow[t]{2}{*}{ Cervus elaphurus scoticus } & Is & & 2.6 & & 14.3 & $1: 0$ & Gyllensten et al. (1983) \\
\hline & M & & 3.3 & & 13.1 & & \\
\hline \multirow[t]{2}{*}{ Lemur macaco } & Is & 3.4 & 3.3 & 1.25 & 25.0 & $1: 0$ & Arnaud et al. (1992) \\
\hline & M & 7.0 & 3.3 & 1.33 & 33.3 & & \\
\hline \multirow[t]{2}{*}{ Macaca fascicularis } & Is & 5.2 & & & 22.7 & $7: 0$ & Kondo et al. (1993) \\
\hline & M & 9.9 & & & 40.2 & & \\
\hline \multirow[t]{2}{*}{ Macaca fuscata } & Is & 1.4 & & 1.05 & 3.6 & $1: 1$ & Nozawa et al. (1975) \\
\hline & M & 1.8 & & 1.15 & 12.9 & & \\
\hline \multirow[t]{2}{*}{ Macaca fuscata } & Is & 0.5 & & & 3.1 & $1: 0$ & Nozawa et al. (1991) \\
\hline & M & 2.3 & & & 14.6 & & \\
\hline \multirow[t]{2}{*}{ Macrotus waterhousii } & Is & 3.0 & 4.0 & 1.19 & 4.8 & $0: 1$ & Greenbaum \& Baker (1976) \\
\hline & M & 2.7 & 2.1 & 1.16 & 7.9 & & \\
\hline \multirow{2}{*}{ Mastomys erytholeucus } & Is & 9.0 & 6.0 & 1.2 & 20.0 & $1: 0$ & Duplantier et al. (1990) \\
\hline & M & 24.6 & 16.2 & 1.42 & 39.4 & & \\
\hline \multirow[t]{2}{*}{ Mastomys huberti } & Is & 10.0 & 7.0 & 1.25 & 20.0 & 1:0 & Duplantier et al. (1990) \\
\hline & M & 24.4 & 16.2 & 1.58 & 48.0 & & \\
\hline \multirow[t]{2}{*}{ Melomys cervinipes } & Is $\dagger$ & 1.0 & & 1.08 & 8.3 & $1: 0$ & Leung et al. (1993) \\
\hline & M & 5.1 & & 1.21 & 20.8 & & \\
\hline \multirow[t]{2}{*}{ Mus musculus } & Is & & 3.6 & 1.20 & 14.5 & $17: 2$ & Berry \& Peters (1977) \\
\hline & M & & 7.4 & 1.33 & 30.2 & & \\
\hline \multirow[t]{2}{*}{ Mus musculus } & Is & & 7.7 & 1.33 & 36.4 & $0: 1$ & Berry et al. (1978) \\
\hline & M & & 7.4 & 1.33 & $\begin{array}{r}34.9 \\
77\end{array}$ & & \\
\hline Mus musculus & $\begin{array}{l}\text { Is } \\
\text { M }\end{array}$ & & $\begin{array}{l}3.4 \\
7.4\end{array}$ & $\begin{array}{l}1.07 \\
1.33\end{array}$ & & $1: 0$ & Berry et al. (1979) \\
\hline \multirow[t]{2}{*}{ Mus musculus } & Is & & 13.0 & & 38.9 & $0: 3$ & Berry et al. (1981) \\
\hline & M & & 8.2 & & 27.2 & & \\
\hline \multirow{2}{*}{ Mus musculus } & Is & 9.4 & & 1.33 & 25.4 & $2: 3$ & Navajas y Navarro \& \\
\hline & M & 8.8 & & 1.3 & 27.0 & & Britton-Davidian (1989) \\
\hline \multirow[t]{2}{*}{ Panthera leo leo } & Crater & & 2.2 & 1.12 & 10.0 & 1:0 & Packer et al. (1991) \\
\hline & M & & 3.3 & 1.22 & 17.5 & & \\
\hline \multirow[t]{2}{*}{ Panthera pardus } & Is & & 1.4 & & 4.0 & 1:0 & Miththapala et al. (1991) \\
\hline & M & & 3.1 & & 10.0 & & \\
\hline \multirow[t]{2}{*}{ Perameles gunii } & Is & 0.0 & 0.0 & 1.0 & 0.0 & - & Sherwin et al. (1991) \\
\hline & M & 0.0 & 0.0 & 1.0 & 0.0 & & \\
\hline Peromyscus eremicus & Is & & 1.3 & & 8.6 & $2: 0$ & Avise et al. (1974a) \\
\hline Peromyscus gossypinus & $\mathrm{M}$ & & 4.0 & & $\begin{array}{l}11.6 \\
413\end{array}$ & 3.3 & \\
\hline & $\begin{array}{l}\text { Is } \\
\text { M }\end{array}$ & & $\begin{array}{l}9.9 \\
9.9\end{array}$ & $\begin{array}{l}1.7 \\
1.7\end{array}$ & $\begin{array}{l}41.3 \\
38.9\end{array}$ & $3: 3$ & Boone et al. (1993) \\
\hline Peromyscus leucopus & Is & & 6.8 & & 16.7 & $3: 0$ & Browne (1977) \\
\hline & M & & 8.0 & & 22.6 & & \\
\hline Peromyscus maniculatus & Is & & 6.6 & 1.37 & 29.0 & $7: 1$ & Gill (1980) \\
\hline & M & & 8.3 & 1.64 & 43.0 & & \\
\hline Peromyscus maniculatus & Is & & 4.2 & & 16.7 & $6: 0$ & Aquadro \& Kilpatrick (1981) \\
\hline & $\mathbf{M}$ & & 9.6 & & 27.6 & & \\
\hline
\end{tabular}


Table 3 Continued

\begin{tabular}{|c|c|c|c|c|c|c|c|}
\hline Species & Is vs. $M$ & $H_{\mathrm{e}}$ & $H_{\mathrm{o}}$ & $A$ & $P$ & $\begin{array}{l}\text { M > Is: } \\
M<I s^{*}\end{array}$ & Reference \\
\hline Peromyscus polionotus & $\begin{array}{l}\text { Is } \\
\text { M }\end{array}$ & $\begin{array}{l}4.9 \\
6.3\end{array}$ & & & & - & Selander et al. (1971) \\
\hline Peromyscus polionotus & $\begin{array}{l}\text { Is } \\
\mathrm{M}\end{array}$ & & $\begin{array}{l}5.9 \\
5.2\end{array}$ & & & 一 & Garten (1976) \\
\hline Peromyscus polionotus & $\begin{array}{l}\text { Is } \\
\text { M }\end{array}$ & $\begin{array}{l}4.6 \\
9.4\end{array}$ & $\begin{array}{l}3.6 \\
8.6\end{array}$ & $\begin{array}{l}1.12 \\
1.35\end{array}$ & $\begin{array}{l}11.5 \\
27.5\end{array}$ & $2: 0$ & Brewer et al. (1990) \\
\hline Rattus fuscipes & $\begin{array}{l}\text { Is } \\
\text { M }\end{array}$ & & $\begin{array}{l}1.0 \\
5.3\end{array}$ & $\begin{array}{l}1.03 \\
1.18\end{array}$ & $\begin{array}{r}3.1 \\
17.9\end{array}$ & $10: 0$ & Schmitt (1978) \\
\hline Rattus rattus & $\begin{array}{l}\text { Is } \\
\text { M }\end{array}$ & & $\begin{array}{l}2.7 \\
3.1\end{array}$ & & $\begin{array}{r}10.0 \\
8.1\end{array}$ & $5: 3$ & Patton et al. (1975) \\
\hline Sigmodon hispidus & $\begin{array}{l}\text { Is } \\
\text { M }\end{array}$ & & $\begin{array}{l}2.1 \\
2.2\end{array}$ & $\begin{array}{l}1.05 \\
1.09\end{array}$ & $\begin{array}{l}4.5 \\
8.6\end{array}$ & 1:0 & Johnson \& Selander (1972) \\
\hline Sorex cinereus & $\begin{array}{l}\text { Is } \\
\text { M }\end{array}$ & $\begin{array}{l}5.6 \\
7.9\end{array}$ & $\begin{array}{l}5.4 \\
7.8\end{array}$ & $\begin{array}{l}1.22 \\
1.43\end{array}$ & $\begin{array}{l}12.9 \\
15.6\end{array}$ & $4: 1$ & Stewart \& Baker (1992) \\
\hline Spermophilus spilosoma & $\begin{array}{l}\text { Is } \\
\mathrm{M}\end{array}$ & & $\begin{array}{l}0.9 \\
9.0\end{array}$ & & $\begin{array}{r}3.4 \\
30.1\end{array}$ & $1: 0$ & Cothran et al. (1977) \\
\hline Birds & & & & & & $21: 6$ & \\
\hline Aplonis cantoroides & $\begin{array}{l}\text { Is } \\
\text { M }\end{array}$ & $\begin{array}{l}0.9 \\
2.6\end{array}$ & $\begin{array}{l}1.1 \\
2.1\end{array}$ & $\begin{array}{l}1.06 \\
1.06\end{array}$ & $\begin{array}{l}5.6 \\
5.6\end{array}$ & $3: 0$ & Corbin et al. (1974) \\
\hline Aplonis metallica & $\begin{array}{l}\text { Is } \\
\text { M }\end{array}$ & $\begin{array}{l}4.1 \\
4.9\end{array}$ & $\begin{array}{l}4.0 \\
4.9\end{array}$ & $\begin{array}{l}1.19 \\
1.22\end{array}$ & $\begin{array}{l}11.1 \\
11.1\end{array}$ & $4: 1$ & Corbin et al. (1974) \\
\hline Callipepla californica & $\begin{array}{l}\text { Is } \\
\text { M }\end{array}$ & $\begin{array}{l}2.9 \\
4.0\end{array}$ & $\begin{array}{l}2.2 \\
3.4\end{array}$ & $\begin{array}{l}1.16 \\
1.26\end{array}$ & $\begin{array}{l}13.5 \\
18.9\end{array}$ & 1:0 & Zink et al. (1987) \\
\hline Empidonax difficilis & $\begin{array}{l}\text { Is } \\
\mathrm{M}\end{array}$ & $\begin{array}{l}3.4 \\
5.6\end{array}$ & $\begin{array}{l}3.7 \\
5.4\end{array}$ & $\begin{array}{l}1.12 \\
1.29\end{array}$ & $\begin{array}{r}9.8 \\
22.6\end{array}$ & $1: 0$ & Johnson \& Marten (1988) \\
\hline Fringilla coelebs & $\begin{array}{l}\text { Is } \\
\text { M }\end{array}$ & $\begin{array}{l}4.3 \\
5.2\end{array}$ & & $\begin{array}{l}1.21 \\
1.34\end{array}$ & $\begin{array}{l}18.7 \\
24.3\end{array}$ & $9: 5$ & Baker et al. (1990) \\
\hline Lagopus lagopus & $\begin{array}{l}\text { Is } \\
\text { M }\end{array}$ & & $\begin{array}{l}6.9 \\
8.0\end{array}$ & & $\begin{array}{l}20.3 \\
26.0\end{array}$ & $3: 0$ & Gyllensten et al. (1985) \\
\hline Reptiles & & & & & & $20: 6$ & \\
\hline Anolis carolinensis & $\begin{array}{l}\text { Is } \\
\text { M }\end{array}$ & & $\begin{array}{l}6.0 \\
5.7\end{array}$ & & $\begin{array}{l}17.0 \\
13.9\end{array}$ & $0: 1$ & Webster et al. (1972) \\
\hline Lacerta sicula & $\begin{array}{l}\text { Is } \\
\text { M }\end{array}$ & & $\begin{array}{l}4.4 \\
9.0\end{array}$ & & $\begin{array}{l}16.8 \\
36.3\end{array}$ & $4: 0$ & Gorman et al. (1975) \\
\hline Trachydosaurus rugosus & $\begin{array}{l}\text { Is } \\
\text { M }\end{array}$ & $\begin{array}{l}12.3 \\
16.1\end{array}$ & $\begin{array}{l}11.9 \\
14.3\end{array}$ & $\begin{array}{l}1.33 \\
1.62\end{array}$ & $\begin{array}{l}29.5 \\
36.1\end{array}$ & $7: 0$ & Sarre et al. (1990) \\
\hline Uta stansburiana & $\begin{array}{l}\text { Is } \\
\text { M }\end{array}$ & & $\begin{array}{l}4.9 \\
5.3\end{array}$ & & $\begin{array}{l}26.2 \\
28.7\end{array}$ & $9: 5$ & $\begin{array}{l}\text { Soulé \& Yang (1973) } \\
\text { McKinney et al. }(1972)\end{array}$ \\
\hline Fish & & & & & & 3:0 & \\
\hline Astyanax mexicanus & $\begin{array}{l}\text { Cave } \\
\text { Surface }\end{array}$ & & $\begin{array}{r}3.6 \\
11.2\end{array}$ & $\begin{array}{l}1.22 \\
2.13\end{array}$ & $\begin{array}{l}13.7 \\
37.3\end{array}$ & $3: 0$ & Avise \& Selander (1972) \\
\hline $\begin{array}{l}\text { Amphibians } \\
\text { Bufo terrestris americanus }\end{array}$ & $\begin{array}{l}\text { Is } \\
\text { M }\end{array}$ & & Is $<\mathrm{M} \S$ & & & $2: 0$ & Abramoff et al. (1964) \\
\hline Bufo viridis & $\begin{array}{l}\text { Is } \\
\mathrm{M}\end{array}$ & & $\begin{array}{r}2.9 \\
13.3\end{array}$ & $\begin{array}{l}1.12 \\
1.65\end{array}$ & $\begin{array}{l}12.0 \\
42.3\end{array}$ & $1: 0$ & Dessauer et al. (1975) \\
\hline Bufo woodhousii fowleri & $\begin{array}{l}\text { Is } \\
\text { M }\end{array}$ & $\begin{array}{l}1.1 \\
3.0\end{array}$ & & $\begin{array}{l}1.14 \\
1.14\end{array}$ & $\begin{array}{l}14.3 \\
14.3\end{array}$ & $1: 0$ & Hranitz et al. (1993) \\
\hline $\begin{array}{l}\text { Molluscs } \\
\text { Cerion bendalli }\end{array}$ & $\begin{array}{l}\text { Is } \\
\text { M }\end{array}$ & & $\begin{array}{l}4.8 \\
5.4\end{array}$ & & $\begin{array}{l}14.3 \\
19.0\end{array}$ & $\begin{array}{l}2: 0 \\
2: 0\end{array}$ & Woodruff (1975) \\
\hline
\end{tabular}


Table 3 Continued

\begin{tabular}{|c|c|c|c|c|c|c|c|}
\hline Species & Is vs. $\mathrm{M}$ & $H_{\mathrm{e}}$ & $H_{\mathrm{o}}$ & $A$ & $P$ & $\begin{array}{l}\text { M > Is: } \\
\text { M }<\text { Is* }\end{array}$ & Reference \\
\hline Insects & & & & & & $23: 4$ & \\
\hline \multirow[t]{2}{*}{ Drosophila equinoxialis caribbensis } & Is & & 17.1 & & 45.1 & $5: 0$ & Ayala et al. (1974) \\
\hline & M & & 22.2 & & 60.9 & & \\
\hline \multirow[t]{2}{*}{ Drosophila immigrans } & Is & & 11.5 & 2.24 & 70.6 & $0: 1$ & Steiner et al. (1976) \\
\hline & M & & 9.3 & 1.82 & 52.9 & & \\
\hline \multirow[t]{2}{*}{ Drosophila nebulosa } & Is & 17.0 & & & & $1: 0$ & Powell (1975) \\
\hline & M & 18.8 & & & & & \\
\hline \multirow[t]{2}{*}{ Drosophila simulans } & Is & & 7.3 & 1.46 & 36.6 & $2: 0$ & Steiner et al. (1976) \\
\hline & M & & 16.2 & 2.17 & 55.6 & & \\
\hline \multirow[t]{2}{*}{ Drosophila subobscura } & Is & 14.8 & & & & $4: 1$ & Cabrera et al. (1980) \\
\hline & M & 18.3 & & & & & \\
\hline \multirow{2}{*}{ Drosophila tropicalis } & Is & 16.7 & & & & $1: 0$ & Powell (1975) \\
\hline & M & 19.8 & & & & & \\
\hline \multirow[t]{2}{*}{ Drosophila willistoni } & Is & & 16.2 & & 48.8 & $5: 1$ & Ayala et al. (1971) \\
\hline & M & & 18.4 & & 54.2 & & \\
\hline \multirow[t]{2}{*}{ Philaenus spumarius } & Is & & 6.2 & 1.52 & 35.7 & $5: 1$ & Saura et al. (1973) \\
\hline & M & & 8.7 & 1.86 & 50.0 & & \\
\hline Plants & & & & & & $12: 1$ & \\
\hline \multirow{2}{*}{ Atherosperma moschatum } & Is & 26.1 & 10.2 & 1.83 & 62.8 & $2: 0$ & Shapcott (1994) \\
\hline & M & 28.6 & 8.1 & 2.24 & 78.0 & & \\
\hline \multirow[t]{2}{*}{ Campanula punctata } & Is & & 4.3 & 1.30 & 26.4 & $6: 0$ & Inoue \& Kawahara (1990) \\
\hline & M & & 11.7 & 1.70 & 46.8 & & \\
\hline \multirow[t]{2}{*}{ Cryptomeria japonica } & $\mathrm{M} \ddagger$ & 17.8 & 15.3 & 2.38 & 47.4 & - & Tsumura \& Ohba (1993) \\
\hline & Is & 19.5 & 18.6 & 2.60 & 47.6 & & \\
\hline \multirow[t]{2}{*}{ Eichhornia paniculata } & Is & 3.0 & 2.0 & 1.07 & 7.6 & $1: 0$ & Glover \& Barrett (1987) \\
\hline & M & 9.0 & 7.8 & 1.27 & 23.8 & & \\
\hline \multirow[t]{2}{*}{ Pinus banksiana } & Is $\dagger$ & 15.2 & 16.4 & 2.4 & 59.0 & $2: 0$ & Gauthier et al. (1992) \\
\hline & $\mathrm{M}$ & 16.4 & 17.9 & 2.2 & 61.4 & & \\
\hline \multirow[t]{2}{*}{ Pinus resinosa } & Is & 0.0 & 0.0 & 1.0 & 0.0 & - & Mosseler et al. (1991) \\
\hline & M & 0.0 & 0.0 & 1.0 & 0.0 & & \\
\hline \multirow[t]{2}{*}{ Pinus torreyana } & Is & 0.0 & 0.0 & 1.0 & 0.0 & - & Ledig \& Conkle (1983) \\
\hline & M & 0.0 & 0.0 & 1.0 & 0.0 & & \\
\hline \multirow[t]{2}{*}{ Quercus petraea } & Is & & 39.8 & 3.09 & & $0: 1$ & Zanetto \& Kremer (1995) \\
\hline & M & & 38.0 & 3.22 & & & \\
\hline \multirow[t]{2}{*}{ Turnera ulmifolia } & Is & 4.0 & 7.0 & 1.20 & 20.0 & $1: 0$ & Barrett \& Husband (1989) \\
\hline & M & 12.0 & 11.0 & 1.51 & 46.0 & & \\
\hline Overall & & & & & & $165: 37$ & \\
\hline
\end{tabular}

*Ratio of cases where mainland populations have higher vs. lower genetic variation than island populations, based on $H_{\mathrm{e}}$, or $H_{\mathrm{o}}$.

$\dagger$ Islands in lakes.

¥island population larger than mainland, and source for it after last glacial.

§Quantitative data not reported.

\section{Results}

\section{Nonendemic species}

Comparisons of genetic variation in island and mainland populations of the same species are given in Table 3. In a highly significant majority of cases (165:37) mainland populations had higher heterozygosities than island populations of the same species $\left(\chi_{1}^{2}=81.1, P<0.00005\right)$, the mean reduction being 29 per cent. Allelic diversity and percentage polymorphism also showed higher genetic variation of mainland than island populations. Mammals, birds, reptiles, insects and plants all showed significantly higher levels of genetic variation in mainland than island populations. Other groups showed similar trends, but had few data. If studies are only counted 
Table 4 Genetic and phenotypic variation in island (Is) and mainland (M) populations. Genetic variation was measured using DNA fingerprints, RAPDs, RFLPs, microsatellites and mitochondrial DNA

\begin{tabular}{|c|c|c|c|c|}
\hline Species & Island & Mainland & $\begin{array}{l}M>\text { Is: } \\
M<\text { Is }\end{array}$ & Reference \\
\hline \multicolumn{5}{|c|}{ DNA fingerprints, RAPDs and microsatellites } \\
\hline Canis lupus* & 28.5 & 68.5 & $1: 0$ & Wayne et al. (1991b) \\
\hline Perameles gunii* & 24.4 & 38.8 & 1:0 & Robinson et al. (1993) \\
\hline Urocyon littoralis* & 12.0 & 52.9 & $6: 0$ & Wayne et al. (1991a) \\
\hline Ursus americanus $\ddagger$ & 36.0 & 79.2 & $1: 0$ & Paetkau \& Strobeck (1994) \\
\hline Insects & & & $3: 0$ & \\
\hline Drosophila subobscura $\S$ & 0.00278 & 0.00562 & $1: 0$ & Rozas \& Aguadé (1991) \\
\hline Nicrophorus americanus $\dagger$ & 96.0 & 92.0 & 1:0 & Kozol et al. (1994) \\
\hline Nicrophorus orbicollis $\dagger$ & 79.5 & 77.1 & $1: 0$ & Kozol et al. (1994) \\
\hline \multicolumn{5}{|l|}{ Mitochondrial DNA } \\
\hline Mammals & & & $16: 0$ & \\
\hline Canis lupus $\mathbb{I}$ & 1 & 9 & 1:0 & Wayne et al. (1991b) \\
\hline Perameles gunii ${ }^{* *}$ & 0.50 & 0.61 & $1: 0$ & Robinson (1995) \\
\hline Peromyscus maniculatus ** & 0.20 & 0.79 & $8: 0$ & Ashley \& Wills (1987) \\
\hline Urocyon littoralis $* *$ & 0.027 & 0.162 & $6: 0$ & Wayne et al. (1991a) \\
\hline \multicolumn{3}{|l|}{ Insects } & $1: 0$ & \\
\hline Drosophila sechellia** & 0.00036 & $0.0020 \dagger \dagger$ & $1: 0$ & Cariou et al. (1990) \\
\hline Drosophila simulans & Is $<$ M枺 & & & Hale \& Singh (1991) \\
\hline \multirow{2}{*}{\multicolumn{3}{|c|}{$\begin{array}{l}\text { Phenotypic variation } \\
\text { Reptiles }\end{array}$}} & $24: 0$ & \\
\hline & & & & \\
\hline Urocyon littoralis & 0.016 & 0.022 & 6:0 & Wayne et al. (1991a) \\
\hline Uta stansburiana & 7.16 & 8.83 & $18: 0$ & Soulé (1972) \\
\hline
\end{tabular}

*Average percent difference for DNA fingerprints.

$\dagger$ Percentage of band sharing for RAPDs.

$¥$ Microsatellites heterozygosity.

§RFLP nucleotide diversity.

INumber of mt DNA haplotypes.

**Nucleotide diversity.

$\dagger$ †rosophila melanogaster.

¥Quantitative data not reported.

once, 48 of 57 comparisons showed higher genetic variation in mainland than island populations with one tie $\left(\chi_{1}^{2}=28.1, P<0.00005\right)$. Nuclear DNA variability (DNA fingerprint, RAPDs and microsatellites) was lower in island populations than in mainland populations in all 12 comparisons $\left(\chi_{1}^{2}=12.0\right.$, $P=0.00025$; Table 4). Mitochondrial DNA nucleotide diversity was lower in island populations than in mainland populations (Table 4) in all 17 comparisons $\left(\chi_{1}^{2}=17.0, P<0.00005\right)$. The phenotypic coefficient of variation was higher in mainland populations than in island populations (Table 4$)$ in all 24 comparisons $\left(\chi_{\mathrm{i}}^{2}=24.0, P<0.00005\right)$.

Taxa did not differ significantly in the reduction in heterozygosity (Kruskal-Wallis $H=13.76$, d.f. $=7$, $P=0.057$; Table 5). However, species capable
Table 5 Ratios of heterozygosities in island populations to mainland populations $\left(H_{\mathrm{I}} \mathrm{s} / H_{\mathrm{M}}\right)$ in different taxa for nonendemic species and for endemic island species compared to related mainland species, and the sample sizes $(N)$

\begin{tabular}{lcrlrr}
\hline & \multicolumn{2}{c}{ Nonendemics } & & \multicolumn{2}{c}{ Endemics } \\
\cline { 2 - 3 } \cline { 5 - 6 } Taxon & $H_{\mathrm{Is}} / H_{\mathrm{M}}$ & $N$ & & $H_{\mathrm{l}} / H_{\mathrm{M}}$ & $N$ \\
\hline Mammals & 0.65 & 102 & & 0.20 & 10 \\
Birds & 0.79 & 27 & 0.37 & 9 \\
Reptiles & 0.78 & 26 & & \\
Fish & 0.32 & 3 & & \\
Amphibians & 0.24 & 2 & & \\
Molluscs & 0.88 & 2 & & \\
Insects & 0.79 & 27 & 0.83 & 8 \\
Arachnids & & & 0.47 & 1 \\
Plants & 0.71 & 13 & 0.54 & 10 \\
\hline
\end{tabular}

(c) The Genetical Society of Great Britain, Heredity, 78, 311-327. 
Table 6 Allozyme heterozygosity of endemic island (Is) species as compared to related mainland (M) species

\begin{tabular}{|c|c|c|c|c|}
\hline \multicolumn{2}{|l|}{$\begin{array}{l}\text { Island species } \\
\text { Mainland species }\end{array}$} & \multirow[t]{2}{*}{ Heterozygosity } & \multirow{2}{*}{$\begin{array}{c}\text { M>Is: } \\
M<\text { Is }\end{array}$} & \multirow[t]{2}{*}{ Reference } \\
\hline Mammals & & & & \\
\hline Dipodomys compactus & Is & 0.6 & $1: 0$ & Johnson \& Selander (1971) \\
\hline Dipodomys & M & 2.1 & & \\
\hline Macaca fuscata & Is & 3.2 & $1: 0$ & Nozawa et al. (1991) \\
\hline Macaca mulatta & M & 7.4 & & \\
\hline Microtus breweri & Is & 0.7 & $1: 0$ & Kohn \& Tamarin (1978) \\
\hline Microtus pennsylvanicus & $\mathrm{M}$ & 6.0 & & \\
\hline Peromyscus eva & Is & 0.0 & 1:0 & Avise et al. (1974a) \\
\hline Haplomys & M & 3.0 & & \\
\hline Peromyscus diskeyi & Is & 0.0 & $1: 0$ & Avise et al. (1974a) \\
\hline Haplomys & M & 3.0 & & \\
\hline Peromyscus guardia & Is & 1.4 & 1:0 & Avise et al. (1974a) \\
\hline Haplomys & M & 3.0 & & \\
\hline Peromyscus interparietalis & Is & 0.0 & 1:0 & Avise et al. (1974a) \\
\hline Haplomys & M & 3.0 & & \\
\hline Peromyscus sejugis & Is & 1.7 & $1: 0$ & Avise et al. $(1974 \mathrm{a}, 1979)$ \\
\hline maniculatis species group & M & 8.1 & & Selander et al. (1971), Kilpatrick (1981) \\
\hline Peromyscus stephani & Is & 0.0 & $1: 0$ & Avise et al. $(1974 \mathrm{a}, \mathrm{b})$ \\
\hline boylii species group & M & 3.2 & & Kilpatrick \& Zimmerman (1975) \\
\hline Urocyon littoralis & Is & 2.0 & $1: 0$ & Wayne et al. (1991a) \\
\hline Urocyon cinereoargenteus & M & 9.7 & & \\
\hline Birds & & & $9: 0$ & \\
\hline Hemignathus parvus & Is & 1.4 & $1: 0$ & Johnson et al. (1989) \\
\hline Family Emberizidae & M & 5.6 & & \\
\hline Hemignathus virens* & Is & 4.8 & 1:0 & Johnson et al. (1989) \\
\hline Himatione sanguinea* & Is & 2.0 & $1: 0$ & Johnson et al. (1989) \\
\hline Loxioides bailleui* & Is & 0.0 & $1 ; 0$ & Johnson et al. (1989) \\
\hline Loxops coccineus* & Is & 0.0 & $1: 0$ & Johnson et al. (1989) \\
\hline Oreomystis bairdi* & Is & 2.8 & $1: 0$ & Johnson et al. (1989) \\
\hline Paroreomyza montana* & Is & 4.9 & $1: 0$ & Johnson et al. (1989) \\
\hline Telespiza cantans* & Is & 0.7 & $1: 0$ & Johnson et al. (1989) \\
\hline Vestiaria coccinea* & Is & 2.0 & $1: 0$ & Johnson et al. (1989) \\
\hline Insects & & & $5: 3$ & \\
\hline Drosophila adiostola & Is & 14.0 & $1: 0$ & Ayala (1975) \\
\hline Drosophila willistoni group & M & 20.2 & & Powell (1975) \\
\hline Drosophila crassifemur $\dagger$ & Is & 20.3 & $0: 1$ & Ayala (1975) \\
\hline Drosophila dolichotarisi $\dagger$ & Is & 13.8 & $1: 0$ & Ayala (1975) \\
\hline Drosophila nigella $\dagger$ & Is & 15.0 & $1: 0$ & Ayala (1975) \\
\hline Drosophila nigra $\dagger$ & Is & 16.0 & $1: 0$ & Ayala (1975) \\
\hline Drosophila planitibia $\dagger$ & Is & 23.6 & $0: 1$ & Ayala (1975) \\
\hline Drosophila sechellia & Is & 2.7 & $1: 0$ & Cariou et al. (1990) \\
\hline Drosophila melanogaster & M & 11.7 & & \\
\hline Drosophila truncipenna $\dagger$ & Is & 22.4 & $0: 1$ & Ayala (1975) \\
\hline Arachnids & & & $1: 0$ & \\
\hline Steganacarus $(S$.$) tenerifensis$ & Is & 2.6 & $1: 0$ & Avanzati et al. (1994) \\
\hline Steganacarus (S.) magnus \& S. (S.) hirsutus & M & 5.6 & & \\
\hline
\end{tabular}


Table 6 Continued

\begin{tabular}{|c|c|c|c|c|}
\hline \multicolumn{2}{|l|}{$\begin{array}{l}\text { Island species } \\
\text { Mainland species }\end{array}$} & Heterozygosity & $\begin{array}{l}\mathrm{M}>\mathrm{Is}: \\
\mathrm{M}<\mathrm{IS}\end{array}$ & Reference \\
\hline Plants & & & $9: 1$ & \\
\hline Crepidiastrum ameristophyllum & Is & 5.1 & $1: 0$ & Ito \& Ono (1990) \\
\hline Crepidiastrum \& Youngia sp. & M & 8.2 & & \\
\hline Crepidiastrum grandicollum & Is & 2.0 & $1: 0$ & Ito \& Ono (1990) \\
\hline Crepidiastrum \& Youngia sp. & $\mathbf{M}$ & 8.2 & & \\
\hline Crepidiastrum linguaefolium & Is & 2.0 & $1: 0$ & Ito \& Ono (1990) \\
\hline Crepidiastrum \& Youngia sp. & M & 8.2 & & \\
\hline Galvezia leucantha & Is & 1.1 & $1: 0$ & Elisens (1992) \\
\hline Galvezia fruticosa & M & 6.8 & & \\
\hline Gossypium darwinii & Is & 3.2 & $1: 0$ & Wendel \& Percy (1990) \\
\hline Gossypium barbadense & $\mathbf{M}$ & 6.7 & & Percy \& Wendel (1990) \\
\hline Gossypium klotzschianum & Is & 3.4 & $1: 0$ & Wendel \& Percival (1990) \\
\hline Gossypium davidsonii & M & 8.7 & & \\
\hline Gossypium tomentosum & Is & 3.3 & $1: 0$ & DeJoode \& Wendel (1992) \\
\hline Gossypium hirsutum & M & 10.8 & & Wendel et al. (1992) \\
\hline Hosta jonesii & Is & 27.3 & $0: 1$ & Chung (1994) \\
\hline Hosta capitata & $\mathrm{M}$ & 15.3 & & \\
\hline Rhaphithamnus venusta & Is & 2.8 & $1: 0$ & Crawford et al. (1993) \\
\hline Rhaphithamnus spinosus & $\mathbf{M}$ & 10.3 & & \\
\hline Solanum fernandezianum & Is & 0.0 & $1: 0$ & Spooner et al. (1992) \\
\hline S. etuberosum \& S. brevidens & M & 10.3 & & \\
\hline Overall & & & $34: 4$ & \\
\hline
\end{tabular}

*The mainland comparison was family Emberizidae as for Hemignathus parus above.

$\dagger$ The mainland comparison was with the Drosophila willistoni group, as for Drosophila adiostola above.

of flight (bats, birds and insects) showed proportionately less reduction in heterozygosity than those that cannot fly (Kruskal-Wallis $H=6.4$, d.f. $=1$, $P=0.006)$.

\section{Endemic species}

Insular endemic species showed lower genetic variation than related mainland species in 34 of 38 cases (Table $6 ; \chi_{1}^{2}=23.7, P<0.00005$ ). Taxa differed significantly in the reduction in heterozygosity (KruskalWallis $H=13.6$, d.f. $=4, P=0.009$ ). The proportionate reduction was greatest in mammals, intermediate in birds, arachnids and plants, and least in insects (Table 5).

\section{Comparison of endemic and nonendemic species}

The ratio of allozyme genetic variation in island/ mainland for endemic species was proportionately lower than that for nonendemic populations in taxa combined (Mann-Whitney $W=25920.5$, $P<0.00005$ ), mammals (Mann-Whitney $W=6106$, $P=0.00025$ ) and birds (Mann-Whitney $W=578$,
$P=0.0022)$ and plants showed a similar trend (Mann-Whitney $W=175, \quad P=0.13$ ); conversely, insects showed no evidence of such a difference (Table 5). The difference in birds remained significant when the mean of all potential mainland relatives was used for comparisons $(W=565, P=0.009)$.

\section{Discussion}

The major findings of this study are that a significant majority of island populations have lower levels of genetic variation than corresponding mainland populations, and that insular endemic species show proportionately lower genetic variation than nonendemic species. The former conclusion is based on allozymes, mtDNA, and nuclear DNA, though most evidence was for allozymes. Phenotypic variation was lower in island than mainland populations, so quantitative genetic variation is probably lower in island populations. Further, inversion polymorphism is lower in island than continental populations (Ayala \& Campbell, 1974). DeJoode \& Wendel (1992) reported that allozyme heterozygosity in over 60 insular endemic plant taxa was 43 per cent of that 
in plants generally, or 67 per cent of that in all endemic (mostly mainland) plants. The equivocal nature of previous conclusions (see Introduction) can be attributed to small sample sizes.

In a similar vein, introduced populations typically have lower levels of genetic variation than native ones within several species of lizards (Taylor \& Gorman, 1975; Gorman et al., 1978; Capula, 1994), land snails (Selander \& Kaufman, 1973; Johnson, 1988), insects (Bryant et al., 1981; Gasperi et al., 1991) and plants (Schwaegerle \& Schaal, 1979; Clegg \& Brown, 1983; Barrett \& Husband, 1989; Novak \& Mack, 1993). Stone \& Sunnucks (1993) found a pattern of reducing genetic variation with founder effects as a gall wasp spread across Europe.

Differences between island and mainland populations are predicted to be greater for small than large islands, for species with lower dispersal rates, and for distant than near islands (Jaenike, 1973). There is evidence to support each of these predictions. Genetic variation is correlated with island size (Frankham, 1996). Species with lower dispersal ability have been shown to have greater differences from analyses within this paper. Effects of distance from the mainland or a large source population have been detected in lizards (Soulé \& Yang, 1973; Gorman et al., 1975) and in several species of mammals (Kilpatrick, 1981; Schmitt et al., 1995).

Why do some island populations have more genetic variation than mainland populations? Chance, high migration rates, and separate migrations from differentiated mainland populations can all cause this. Notably, a number of island populations of house mice have more genetic variation than mainland populations. House mice have excellent dispersal ability, as indicated by the number of new worldwide locations they have colonized. Further, several subspecies exist and Japanese populations are known to involve mixtures of them (Bonhomme et al., 1989). Pacific island populations on major trade routes (such as on Hawaii) are likely to have had introductions from Europe, Asia, North and South America. Other species where island populations have greater variation than mainland populations are often characterized by high dispersal ability [bat (Macrotus) Greenbaum \& Baker, 1976; moose Ryman et al., 1980; oak (Quercus petraea) Zanneto \& Kremer, 1995]. The plant Cryptomeria japonica is a particularly informative exception (Tsumura \& Ohba, 1993). The island population acted as the refuge during a glacial period, and the smaller mainland population was derived from it. As predicted, the island population has higher genetic variation than the mainland population. Further, the endemic island plant Hosta jonesii, that has a higher population size than the mainland $H$. capitata, has more genetic variation (Chung, 1994). The only other endemics that had higher genetic variation than mainland populations were all Hawaiian Drosophila. These have particularly high levels of genetic variation, especially as they are considered to suffer bottlenecks each time they migrate to a new island and speciate (Carson, 1970; Giddings et al., 1989). The scenario of a single inseminated female founding populations on new islands seems improbable in the light of the allozyme data.

There are four genetic factors that can contribute to higher extinction rates of island compared with mainland populations, namely inbreeding depression, loss of genetic variation, accumulation of mildly deleterious mutations, and genetic adaptations to island environments (flightlessness, limited ability to avoid predators and diseases). The last of these is widely acknowledged as a reason for the extinction proneness of island endemic species (Carlquist, 1974; Myers, 1979; Soulé, 1983; Temple, 1986; Vitousek, 1988; Atkinson, 1989; World Conservation Monitoring Centre, 1992). The evidence herein shows that insular populations, especially endemic species, have their evolutionary potential seriously compromised. Consequently, they are expected to be have limited ability to adapt genetically to environmental change, whether that be newly introduced diseases, global climate change, or introduced predators, or competitors. The susceptibility of Hawaiian birds to introduced avian malaria (Warner, 1968) may be an example of this compromised evolutionary potential. Several genetic mechanisms to resist malaria are known in humans (Allison, 1956; Cavalli-Sforza \& Bodmer, 1971; Ruwende et al., 1995). On genetic grounds, endemic island species are predicted to have higher extinction rates than nonendemic island populations. This has been documented for New Zealand land birds (McDowall, 1969), and for reptiles (Case et al., 1992). It probably applies to most other taxa, but I am unaware of data on comparative extinction risks for them. Inbreeding depression increases the risk of extinction (Soulé, 1980; Frankham, 1995b), but is rarely mentioned as a cause of high extinction rates of island populations. This issue is worthy of detailed consideration. Arguments about whether demographic, environmental or genetic stochasticity cause extinctions are misguided. Genetic threats to island populations will interact with other threats to increase the risk of extinction of island populations.

In conclusion, an overwhelming majority of island populations have lower genetic variation than their 
mainland counterparts, with endemic island species being proportionately lower than nonendemic species. Consequently, the hypothesis that genetic factors contribute to the higher extinction rate of island than mainland populations cannot be rejected.

\section{Acknowledgements}

I thank S. C. H. Barrett, R. J. Berry, D. Gilligan, A. Lindsay, E. Nevo, D. Spielman, P. Sunnucks, A. Taylor and L. Woodworth for comments on the manuscript, E. A. Thompson for statistical advice, and $\mathrm{H}$. Manning and the Interlibrary Loan section of the Macquarie University Library for assistance in obtaining references. My research is supported by Australian Research Council and Macquarie University research grants. Publication no. 196 of the Key Centre for Biodiversity and Bioresources.

\section{References}

ABRAMOFF, P., DARNELl, R. M. AND BALSANO, J. S. 1964. Serological relations of the toad populations of the Lake Michigan area. In: Leone, C. A. (ed.) Taxonomic Biochemistry and Serology, pp. 512-525. Ronald Press, New York.

Allison, A. C. 1956. The sickle-cell and haemoglobin C genes in some African populations. Ann. Hum. Genet., 21, 67-89.

AQUADRo, C. F. AND KILPATRICK, c. w. 1981. Morphological and biochemical variation and differentiation in insular and mainland deer mice (Peromyscus maniculatus). In: Smith, M. H. and Joule, J. (eds) Mammalian Population Genetics, pp. 214-230. University of Georgia Press, Athens, GA.

ARNAUD, J., MEIER, B., DUGOUJON, J. M. AND RUMPLER, Y. 1992. Study of the variability of erythrocyte enzymes in captive and wild populations of the black lemur (Eulemur macaco macaco). An indispensable preliminary to captive breeding programs. Primates, 33, 139-146.

ASHLEY, M. AND wILLs, C. 1987. Analysis of mitochondrial DNA polymorphisms among Channel Island deer mice. Evolution, 41, 854-863.

ATKINSON, I, 1989. Introduced animals and extinction. In: Western, D. and Pearl, M. C. (eds) Conservation for the Twenty-First Century, pp. 54-75. Oxford University Press, New York.

AVANZATI, A. M., BARATTI, M. AND BERNINI, F. 1994. Molecular and morphological differentiation between steganacarid mites (Acari: Oribatida) from the Canary islands. Biol. J. Linn. Soc., 52, 325-340.

AVISE, J. C. AND SELANDER, R. K. 1972. Evolutionary genetics of cave-dwelling fishes of the genus Astyanax. Evolution, 26, 1-19.

AVISE, J. C., SMITH, M. H. AND SELANDER, R. K. 1974a.
Biochemical polymorphism and systematics in the genus Peromyscus. VI. The boylii species group. $J$. Mammal, 55, 751-763.

AVISE, J. C., SMITH, M. H., SELANDER, R. K., LAWLOR, T. E. AND RAMSEY, P. R. 1974b. Biochemical polymorphism and systematics in the genus Peromyscus. V. Insular and mainland species of the subgenus Haplomys. Syst. Zool., 23, 226-238.

AVISE, J. C., SMITH, M. H. AND SELANDER, R. K. 1979. Biochemical polymorphism and systematics in the genus Peromyscus. VIII. Geographic differentiation in members of the truei and maniculatus species group. $J$. Mammal., 60, 177-192.

AYALA, F. J. 1965. Evolution of fitness in experimental populations of Drosophila serrata. Science, 150, 903-905.

AYALA, F, J. 1975. Genetic differentiation during the speciation process. In: Dobzhansky, T., Hecht, M. K. and Steere, W. C. (eds) Evolutionary Biology, vol. 8, pp. 1-78. Plenum Press, New York.

AYALA, F. J. AND CAMPBELL, C. A. 1974. Frequency-dependent selection. Ann. Rev. Ecol. Syst., 5, 115-138.

AYALA, F. J., POWELl, J. R. AND DOBZHANSKY, T. 1971. Polymorphisms in continental and island populations of Drosophila willistoni. Proc. Natl. Acad. Sci. US.A., 68, 2480-2483.

AYALA, F. J., TRACEY, M. L., BARR, L. G. AND EHRENFELD, J. G. 1974. Genetic and reproductive differentiation of the subspecies, Drosophila equinoxialis caribbensis. Evolution, 28, 24-41.

BAKER, A. J., DENNISON, M. D., LYNCH, A. AND LE GRAND, G. 1990. Genetic divergence in peripherally isolated populations of chaffinches in the Atlantic islands. Evolution, 44, 981-999.

BARRETT, S. C. H. AND HUSBAND, B. C. 1989. The genetics of plant migration and colonization. In: Brown, A. H. D., Clegg, M. T., Kahler, A. L. and Weir, B. S. (eds) Plant Population Genetics, Breeding, and Genetic Resources, pp. 254-277. Sinauer Associates, Sunderland, MA.

BERRY, R. J. 1986. Genetics of insular populations of mammals, with particular reference to differentiation and founder effects in British small mammals. Biol. J. Linn. Soc., 28, 205-230.

BERRY, R. J. AND PETERS, J. 1977. Heterogeneous heterozygosities in Mus musculus populations. Proc. R. Soc. B, 197, 485-503.

BERRY, R. J., PETERS, J. AND VAN AARDE, R. J. 1978. Sub-antarctic house mice: colonization, survival and selection. J. Zool. Lond., 184, 127-141.

BERRY, R. J., BONNER, W. N. AND PETERS, J. 1979. Natural selection in house mice (Mus musculus) from South Georgia (South Atlantic Ocean). J. Zool. Lond., 189, 385-398.

BERRY, R. J., SAGE, R. D., LIDICKER, w. Z. AND JACKSON, w. B. 1981. Genetic variation in three Pacific house mouse (Mus musculus) populations. J. Zool. Lond., 193, 391-404.

BOAG, P. T. 1988. The genetics of island birds. In: Ouellet, H. (ed.) Acta XIX International Ornithological Congress, 
pp. 1550-1563. National Museum of Natural Sciences, Ottawa.

BOCK, B. C. AND MCCRACKEN, G. F. 1988. Genetic structure and variability in the green iguana (Iguana iguana). $J$. Herpetol, 22, 316-322.

BONHOMME, F., MIYASHITA, N., BOURSOT, P., CATALAN, 3. AND MORIWAKI, K. 1989. Genetical variation and polyphyletic origin in Japanese Mus musculus. Heredity, 63, 299-308.

BOONE, J. L., LAERM, J. AND SMITH, M. H. 1993. Taxonomic status of Peromyscus gossypinus anastasae (Anastasia Island cotton mouse). J. Mammal., 74, 363-375.

BRAKEFIELD, P. M. AND SACCHERI, I. J. 1994. Guidelines in conservation genetics and the use of population cage experiments with butterflies to investigate the effects of genetic drift and inbreeding. In: Loeschke, V., Tomiuk, J. and Jain, S. K. (eds) Conservation Genetics, pp. 165-179. Birkhäuser Verlag, Basel.

BREWER, B. A., LACY, R. C., FOSTER, M. L. AND ALAKS, G. 1990. Inbreeding depression in insular and central populations of Peromyscus mice. J. Hered., 81, 257-266.

BROWNE, R. A. 1977. Genetic variation in island and mainland populations of Peromyscus leucops. Am. Midl. Nat., 97, 1-9.

BRYANT, E. H., VAN DIJK, H. AND VAN DELDEN, w. 1981. Genetic variability in the face fly Musca autumnalis de Geer, in relation to a population bottleneck. Evolution, 35, 872-881.

BRYANT, E. H., McCOMMAS, S. A. AND COMBS, L. M. 1986. The effect of an experimental bottleneck upon quantitative genetic variation in the housefly. Genetics, 114, 1191-1211.

CABRERA, v. M., GONZÁlez, A. M. AND GULlón, A. 1980. Enzymatic polymorphism in Drosophila subobscura populations from the Canary Islands. Evolution, 34, 875-887.

CAPUlA, M. 1994. Population genetics of a colonizing lizard: loss of variability in introduced populations of Podarcis sicula. Experientia, 50, 691-696.

CARIOU, M.-L., SOlIGNAC, M. AND DAVID, J. R. 1990. Low allozyme and mtDNA variability in the island endemic species Drosophila sechellia (D. melanogaster complex). Experientia, 46, 101-104.

CARLQuist, s. 1974. Island Biology. Columbia University Press, New York.

CARSON, H. L. 1970. Chromosome tracers of the origin of species. Science, 168, 1414-1418.

CASE, T. J., BOLGER, D. T. AND RICHMAN, A. D. 1992. Reptilian extinctions: the last ten thousand years. In: Fiedler, P. L. and Jain, S. K. (eds) Conservation Biology: The Theory and Practice of Nature Conservation, Preservation and Management, pp. 90-125. Chapman and Hall, New York.

CAVALLI-SFORZA, L. L. AND BODMER, w. F. 1971. The Genetics of Human Populations. Freeman, San Francisco.

CHUNG, M. G. 1994. Allozyme diversity and population genetic structure in Hosta jonesii (Liliaceae), a Korean endemic species. Korean J. Genet., 16, 147-156.
CLEGG, M. T. AND BROWN, A. H. D. 1983. The founding of plant populations. In: Schonewald-Cox, C. M., Chambers, S. M., MacBryde, B. and Thomas, L. (eds) Genetics and Conservation: A Reference for Managing Wild Animal and Plant Populations, pp. 216-228. Benjamin/ Cummings, Menlo Park, CA.

CORBIN, K. W., SIBLEY, C. G., FERGUSON, A., WILSON, A. C., BRUSH, A. H. AND AHLOUIST, J. E. 1974. Genetic polymorphism in New Guinea starlings of the genus Aplonis. Condor, 76, 307-318.

COTHRAN, E. G., ZIMMERMAN, E. G. AND NADLER, C. F. 1977. Genic differentiation and evolution in the ground squirrel subgenus Ictidomys (Genus Spermophilus). J. Mammal., 58, 610-622.

CRAWFORD, D. J., STUESSY, T. F., RODRIGUEZ, R. AND RONDINELLI, M. 1993. Genetic diversity in Rhaphithamnus venustus (Verbenaceae), a species endemic to the Juan Fernandez Islands. Bull. Torrey Bot. Club, 120, 23-28.

CROW, J. F. AND KImURA, M. 1970. An Introduction to Population Genetics Theory. Harper and Row, New York.

DAVIS, S. D., DROOP, S. J. M., GREGERSON, P., HENSON, L., LEON, C. J., LAMLEIN VILla-LobOS, J. ET AL. 1986. Plants in Danger: What Do We Do?. International Union for Conservation of Nature and Natural Resources, Gland, Switzerland.

DEJOODE, D. R. AND WENDEL, J. F. 1992. Genetic diversity and the origin of the Hawaiian islands cotton, Gossypium tomentosum. Am. J. Bot., 79, 1311-1319.

DESSAUER, H. C., NEVO, E. AND CHUANG, K.-C. 1975. High genetic variability in an ecologically variable vertebrate, Bufo viridis. Biochem. Genet., 13, 651-661.

DIAMOND, J. M. 1984. Historic extinctions: their mechanisms, and lessons for understanding prehistoric extinctions. In: Martin, P. S. and Klein, R. (eds) Quartenary Extinctions, pp. 824-862. University of Arizona Press, Tucson.

DOBSON, A. P. AND MAY, R. M. 1986. Disease and conservation. In: Soulé, M. E. (ed.) Conservation Biology: The Science of Scarcity and Diversity, pp. 345-365. Sinauer Associates, Sunderland, MA.

DUPLANTIER, J. M., GRANJON, L., MATHIEU, E. AND BONHOMME, F. 1990. Structures génétiques comparées de trois espèces de rongeurs africains du genre Mastomys au Sénégal. Genetica, 81, 179-192.

ELISENS, w. J. 1992. Genetic divergence in Galvezia (Scrophulariaceae): evolutionary and biogeographic relationships among South American and Galápagos species. Am. J. Bot., 79, 198-206.

FEVOLDEN, S. E. 1992. Allozymic variability in the Iceland scallop Chlamys islandica: geographic variation and lack of growth-heterozygosity correlations. Mar. Ecol. Prog. Ser., 85, 259-268.

FLESNESS, N. R. 1989. Mammalian extinction rates: background to the black-footed ferret drama. In: Seal, U. S., Thorne, E. T., Bogan, M. A. and Anderson, S. H. (eds) Conservation Biology of the Black-Footed Ferret, pp. 3-9. Yale University Press, New Haven, CT.

FRANKel, O. H. AND SOULÉ, M. E. 1981. Conservation and 
Evolution. Cambridge University Press, Cambridge.

FRANKHAM, R. 1980. The founder effect and response to artificial selection in Drosophila. In: Robertson, A. (ed.) Selection Experiments in Laboratory and Domestic Animals, pp. 87-90. Commonwealth Agricultural Bureaux, Farnham Royal.

frankham, R. 1995a. Conservation genetics. Ann. Rev. Genet., 29, 305-327.

FRANKHAM, R. 1995b. Inbreeding and extinction: a threshold effect. Conserv. Biol, 9, 792-799.

FRANKHAM, R. 1996. Genetic variation is related to population size in wildlife? Conserv. Biol. (in press).

FRANKLIN, 1. R. 1980. Evolutionary change in small populations. In: Soulé, M. E. and Wilcox, B. A. (eds) Conservation Biology: An Evolutionary-Ecological Perspective, pp. 135-149. Sinauer Associates, Sunderland, MA.

GARTEN, C. T. J. 1976. Relationships between aggressive behavior and genic heterozygosity in the oldfield mouse Peromyscus polionotus. Evolution, 30, 59-72.

GASPERI, G., GUGLIELMINO, C. R., MALACRIDA, A. R. AND MILANI, R. 1991. Genetic variability and gene flow in geographical populations of Ceratitis captitata. Heredity, 67, 347-356.

GAUTHIER, S., SIMON, J.-P. AND BERGERON, Y. 1992. Genetic structure and variability in jack pine populations: effects of insularity. Can. J. Forest Res., 22, 1958-1965.

GIDDINGS, L. V., KANESHIRO, K. Y. AND ANDERSON, W. W. (eds). 1989. Genetics, Speciation and the Founder Principle. Oxford University Press, New York.

GILL, A. E. 1980. Evolutionary genetics of California Island Peromyscus. In: Power, D. M. (ed.) The California Islands: Proceedings of a Multidisciplinary Symposium, pp. 719-743. Santa Barbara Museum of Natural History, Santa Barbara, CA.

GLOVER, D. A. AND BARRETT, S. C. H. 1987. Genetic variation in continental and island populations of Eichhornia paniculata (Pontederiaceae). Heredity, 59, 7-17.

GORMAN, G. C., SOULÉ, M. E., YANG, S. Y. AND NEVO, E. 1975. Evolutionary genetics of insular Adriatic lizards. Evolution, 29, 52-71.

GORMAN, G. C., KIM, Y. J. AND YANG, s. Y. 1978. The genetics of colonization: Loss of variability among introduced populations of Anolis lizards (Reptilia, Lacertilia, Iguanidae). J. Herpetol., 12, 47-51.

GREENBAUM, I. F. AND BAKER, R. J. 1976. Evolutionary relationships in Macrotus (Mammalia: Chiroptera): biochemical variation and karyology. Syst. Zool., 25, 15-25.

GYLLENSTEN, U., RYMAN, N., REUTERWALL, C. AND DRATCH, P. 1983. Genetic differentiation in four European subspecies of red deer (Cervus elaphus L.). Heredity, 51, 561-580.

GYLLENSTEN, U., RYMAN, N. AND SAETHER, T. 1985. Genetic divergence between willow grouse (Lagopus lagopus L.) and rock ptarmigan (Lagopus mutus L.) and the genetic structure of Scandinavian grouse populations. Hereditas, $102,47-55$.

HALE, L. R. AND SINGH, R. S. 1991. Contrasting patterns of genetic structure and evolutionary history as revealed by mitochondrial DNA and nuclear gene-enzyme variation between Drosophila melanogaster and Drosophila simulans. J. Genet., 70, 79-90.

HARTL, G. B. AND PUCEK, z. 1994. Genetic depletion in the European bison (Bison bonasus) and the significance of electrophoretic heterozygosity for conservation. Conserv. Biol., 8, 167-194.

HRANITZ, J. M., KLINGER, T. S., HILL, F. C., SAGAR, R. G., MENCKEN, T. AND CARR, J. 1993. Morphological variation between Bufo woodhousii fowleri Hinckley (Anura: Bufonidae) on Assateague Island, Virginia and the adjacent mainland. Brimleyana, 19, 65-75.

INOUE, K. AND KAWAHARA, T. 1990. Allozyme differentiation and genetic structure in island and mainland Japanese populations of Campanula punctata (Campanulaceae). Am. J. Bot., 77, 1440-1448.

ITO, M. AND ONO, M. 1990. Allozyme diversity and evolution of Crepidiastrum (Compositae) on the Bonin (Ogasawara) Islands. Bot. Mag. Tokyo, 103, 449-459.

JAENIKE, J. R. 1973. A steady state model of genetic polymorphism on islands. Am. Nat., 107, 793-795.

JAMES, J. w. 1971. The founder effect and response to artificial selection. Genet. Res., 16, 241-250.

JOHNSON, M. s. 1988. Founder effects and geographic variation in the land snail Theba pisana. Heredity, 61, 133-142.

JOHNSON, N. K. AND MARTEN, J. A. 1988. Evolutionary genetics of flycatchers. II. Differentiation of the Empidonax difficilis complex. Auk, 105, 177-191.

JOHNSON, N. K., MARTEN, J. A. AND RALPH, C. J. 1989. Genetic evidence for the origin and relationships of Hawaiian honeycreepers (Aves: Fringillidae). Condor, 91, 379-396.

JOHNSON, W. E. AND SELANDER, R. K. 1971. Protein variation and systematics in kangaroo rats (Genus Dipodomys). Syst. Zool., 20, 377-405.

JOHNSON, W. E. AND SELANDER, R. K. 1972. Biochemical genetics of sibling species of the cotton rat (Sigmodon). University of Texas Studies Genet., 7, 297-305.

KILPATRICK, C. w. 1981. Genetic structure of insular populations. In: Smith, M. H. and Joule, J. (eds) Mammalian Population Genetics, pp. 28-59. University of Georgia Press, Athens, GA.

KILPATRICK, C. W. AND ZIMMERMAN, E. G. 1975. Genetic variation and systematics of four species of mice of the Peromyscus boylii species group. Syst. Zool,, 24, 143-162.

KIMURA, M. 1983. The Neutral Theory of Molecular Evolution. Cambridge University Press, Cambridge.

KOHN, P. H. AND TAMARIN, R. H. 1978. Selection at electrophoretic loci for reproductive parameters in island and mainland voles. Evolution, 32, 15-28.

KONDO, M., KaWAMOTO, Y., NOZAWA, K., MATSUbayashl, K., WATANABE, T., GRIFFITHS, O. AND STANLEY, M. 1993. Population genetics of crab-eating macaques (Macaca fascicularis) on the island of Mauritius. Am. J. Primatol., 29, 167-182.

KOZOL, A. J., TRANIELLO, J. F. A. AND WILLIAMS, S. M. 1994. 
Genetic variation in the endangered burying beetle Nicrophorus americanus (Coleoptera: Silphidae). Ann. Entomol. Soc. Am., 87, 928-935.

LEBERG, P. L. 1992. Effects of population bottlenecks on genetic diversity as measured by allozyme electrophoresis. Evolution, 46, 477-494.

LEDIG, F. T. AND CONKLE, M. P. 1983. Gene diversity and genetic structure in a narrow endemic, Torrey pine (Pinus torreyana Parry ex Carr.). Evolution, 37, 79-85.

LEUNG, L. K.-P., DICKMAN, C. R. AND MOORE, L. A. 1993. Genetic variation in fragmented populations of an Australian rainforest rodent, Melomys cervinipes. Pacific Conserv. Biol., 1, 58-65.

LÓPEZ-FANJUL, C. AND VILLAVERDE, A. 1989. Inbreeding increases genetic variance for viability in Drosophila melanogaster. Evolution, 43, 1800-1804.

McDowALL, R. M. 1969. Extinction and endemism in New Zealand land birds. Tuatara, 17, 1-12.

MCKINNEY, C. O., SELANDER, R. K., JOHNSON, W. E. AND YANG, S. Y. 1972. Genetic variation in the side-blotched lizard (Uta stansburiana). In: Studies in Genetics, vol. VII, pp. 307-318. University of Texas Publication no. 7213.

MCNEELY, J. A., MILLER, K. R., REID, W. V., MITTERMEIER, R. A. AND WERnER, T. B. 1990. Conserving the World's Biological Diversity. IUCN, Gland, Switzerland.

MITHTHAPALA, S., SEIDENSTICKER, J., PHILlips, L. G., GOODROWE, K. L., FERNANDO, S. B. U., FORMAN, L. AND O'BRIEN, S. J. 1991. Genetic variation in Sri Lankan leopards. Zoo Biol., 10, 139-146.

MOSSELER, A., INNES, D. J. AND ROBERTS, B. A. 1991. Lack of allozymic variation in disjuct Newfoundland populations of red pine (Pinus resinosa). Can. J. Forest Res., 21, $525-528$.

MYERS, N. 1979. The Sinking Ark. A New Look at the Problem of Disappearing Species. Pergamon Press, New York.

NAVAJAS Y NAVARRO, M. AND BRITTON-DAVIDIAN, J. 1989. Genetic structure of insular Mediterranean populations of the house mouse. Biol. J. Linn. Soc., 36, 377-390.

NEVO, E. 1978. Genetic variation in natural populations: patterns and theory. Theor. Pop. Biol., 13, 121-177.

NEVO, E., BIELES, A. AND BEN-SHLOMO, R. 1984. The evolutionary significance of genetic diversity: ecological, demographic and life history correlates. In: Mani, G. S. (ed.) Evolutionary Dynamics of Genetic Diversity, pp. 13-213. Springer-Verlag, Berlin.

NOVAK, S. J. AND MACK, R. N. 1993. Genetic variation in Bromus tectorum (Poaceae): comparison between native and introduced populations. Heredity, 71, 167-176.

NOZAWA, K., SHOTAKE, T. AND OKURA, Y. 1975. Blood protein polymorphisms and population structure of the Japanese macaque Macaca fuscata fuscata. In: Markert, C. L. (ed.) Isozymes. IV. Genetics and Evolution, pp. 225-241. Academic Press, New York.

NOZAWA, K., SHOTAKE, T., MINEZAWA, M., KaWAMOTO, Y., haYASAKA, K., KaWAMOTO, S. AND ITO, s.-I. 1991. Population genetics of Japanese monkeys: III. Ancestry and differentiation of local populations. Primates, 32, $411-435$.

O'BRIEN, S. J. AND EVERMANN, J. F. 1988. Interactive influence of infectious disease and genetic diversity in natural populations. Trends Ecol. Evol, 3, 254-259.

онтA, т. 1992. The near neutral theory of molecular evolution. Ann. Rev. Ecol. Syst., 23, 263-286.

OLSON, S. L. 1989. Extinction on islands: Man as a catastrophe. In: Western, D. and Pearl, M. C. (eds) Conservation for the Twenty-First Century, pp. 50-53. Oxford University Press, New York.

PACKeR, C., PUSEY, A. E., ROWley, H., GILbERT, D. A., MARTENSON, J. AND O'BRIEN, S. J. 1991. Case study of a population bottleneck: lions of the Ngorongoro crater. Conserv. Biol., 5, 219-230.

PAETKAU, D. AND STROBECK, C. 1994. Microsatellite analysis of genetic variation in black bear populations. Mol. Ecol., 3, 489-495.

PATTON, J. L., YANG, S. Y. AND MYERS, P. 1975. Genetic and morphological divergence among introduced rat populations (Rattus rattus) of the Galapagos archipelago, Ecuador. Syst. Zool., 24, 296-310.

PERCY, T. G. AND WENDEL, J. F. 1990. Allozyme evidence for the origin and diversification of Gossypium barbadense L. Theor. Appl. Genet., 79, 529-542.

PIMM, s. L. 1991. The Balance of Nature: Ecological Issues in the Conservation of Species and Communities. University of Chicago Press, Chicago, IL.

POWELL, J. R. 1975. Protein variation in natural populations of animals. Evol. Biol., 8, 79-119.

REID, w. V. AND MILLER, K. R. 1989. Keeping Options Alive: The Scientific Basis for Conserving Biodiversity. World Resources Institute, Washington, DC.

ROBERTSON, A. 1962. Selection for heterozygotes in small populations. Genetics, 47, 1291-1300.

ROBERTSON, A. 1966. Artificial selection in plants and animals. Proc. R. Soc. B, 164, 341-349.

ROBINSON, N. A. 1995. Implications from mitochondrial DNA for management to conserve the eastern barred bandicoot (Perameles gunii). Conserv. Biol., 9, 114-125.

ROBINSON, N. A., MURRAY, N. D. AND SHERWIN, w. B. 1993. VNTR loci reveal differentiation between and structure within populations of the eastern barred bandicoot Perameles gunnii. Mol. Ecol., 2, 195-207.

ROZAS, J. AND AGUADÉ, M. 1991. Study of an isolated population at the nucleotide level: $r p 49$ region of a Canarian population of Drosophila subobscura. Mol. Biol. Evol., 8, 202-211.

RUWENDE, C., KHOO, S. C., SNOW, R. W., YATES, S. N. R., KWAITKOWSKI, D., GUPTA, S. ET AL. 1995. Natural selection of hemi- and heterozygotes for G6PD deficiency in Africa by resistance to severe malaria. Nature, 376, 246-249.

RYMAN, N., REUTERWALL, C., NYGRÉN, K. AND NYGRÉN, T. 1980. Genetic variation and differentiation in Scandinavian moose (Alces alces): are large mammals monomorphic? Evolution, 34, 1037-1049.

SARRE, S., SCHWANER, T. D. AND GEORGES, A. 1990. Genetic 
variation among insular populations of the sleepy lizard, Trachydosaurus rugosus Gray (Squamata: Scincidae). Aust. J. Zool., 38, 602-616.

SatTa, y., o'HUigin, C., TAKahatA, N. AND KLeIN, J. 1994. Intensity of natural selection at the major histocompatibility complex loci. Proc. Natl. Acad. Sci. U.S.A., 91, 7184-7188.

SAURA, A., HALKKA, O. AND LOKK1, J. 1973. Enzyme gene heterozygosity in small island populations of Philaenus spumarius (L.) (Homoptera). Genetica, 44, 459-473.

SCHMITT, L. H. 1978. Genetic variation in isolated populations of the Australian bush-rat, Rattus fuscipes. Evolution, 32, 1-14.

SCHMITT, L. H., KITCHENER, D. J. AND HOW, R. A. 1995. A genetic perspective of mammalian variation and evolution in the Indonesian archipelago: biogeographic correlates in the fruit bat genus Cynopterus. Evolution, 49, 399-412.

SCHWAEGERLE, K. E. AND SCHAAL, B. A. 1979. Genetic variability and founder effect in the pitcher plant Sarracenia purpurea L. Evolution, 33, 1210-1218.

SELANDER, R. K. 1976. Genetic variation in natural populations. In: Ayala, F. J. (ed.) Molecular Evolution, pp. 21-45. Sinauer Associates, Sunderland, MA.

SELANDER, R. K. AND JOHNSON, W. E. 1973. Genetic variation among vertebrate species. Ann. Rev. Ecol. Syst., 4, 75-91.

SELANDER, R, K. AND KAUfMAN, D. W. 1973. Self-fertilization and genetic population structure in a colonizing land snail. Proc. Natl. Acad. Sci. U.S.A., 70, 1186-1190.

SELANDER, R. K., SMITH, M. H., YANG, S. Y., JOHNSON, W. E. AND GENTRY, J. B. 1971. Biochemical polymorphism and systematics in the genus Peromyscus. I. Variation in the old-field mouse (Peromyscus polionotus). University of Texas Publ. Genet., 7103, 49-90.

SHAPCOTT, A. 1994. Genetic and ecological variation in Atherosperma moschatum and the implications for conservation of its biodiversity. Aust. J. Bot., 42, 663-686.

SHERWIN, W. B., MURRAY, N. D., MARSHALL GRAVES, J. A. AND BROWN, P. R. 1991. Measurement of genetic variation in endangered populations: bandicoots (Marsupialia: Peramelidae) as an example. Conserv. Biol., 5, 103-108.

SMITH, F. D. M., MAY, R. M., PELLEW, R., JOHNSON, T. H. AND WALTER, K, R. 1993. How much do we know about the current extinction rate? Trends Ecol. Evol, 8, 375-378.

SOULÉ, M. E. 1972. Phenetics of natural populations. III. Variation in insular populations of a lizard. Am. Nat., 106, 429-446.

SOULÉ, M. E. 1976. Allozyme variation, its determinants in space and time. In: Ayala, F. J. (ed.) Molecular Evolution, pp. 60-77. Sinauer Associates, Sunderland, MA.

SOULÉ, M. E. 1980. Thresholds for survival: maintaining fitness and evolutionary potential. In: Soulé, M. E. and Wilcox, B. A. (eds) Conservation Biology: An Evolutionary-Ecological Perspective, pp. 151-169. Sinauer Associates, Sunderland, MA.
SOULÉ, M. E. 1983. What do we really know about extinction? In: Schonewald-Cox, C. M., Chambers, S. M., MacBryde, B. and Thomas, L. (eds) Genetics and Conservation: A Reference for Managing Wild Animal and Plant Populations, pp. 111-125. Benjamin/ Cummings, Menlo Park, CA.

SOULÉ, M. E. AND YANG, S. Y. 1973. Genetic variation in side-blotched lizards in the Gulf of California. Evolution, 27, 593-600.

SPOONER, D. M., DOUCHES, D. S. AND CONTRERAS M. A. 1992. Allozyme variation within Solanum Sect. Petota, ser. Etuberosa (Solanaceae). Am. J. Bot., 79, 467-471.

STEINER, W. W. M., SUNG, K. C. AND PAIK, Y. K. 1976. Electrophoretic variability in island populations of Drosophila simulans and Drosophila immigrans. Biochem. Genet., 14, 495-506.

STEPIEN, C. A. AND ROSENBlatT, R. H. 1991. Patterns of gene flow and genetic divergence in the northeastern Pacific Clinidae (Teleosteri: Blennioidei), based on allozymes and morphological data. Copeia, 1991 (4), 873-896.

STEWART, D. T. AND BAKER, A. J. 1992. Genetic differentiation and biogeography of the masked shrew in Atlantic Canada. Can. J. Zool., 70, 106-114.

STONE, G. N. AND SUNNUCKS, P. 1993. Genetic consequences of an invasion through a patchy environment - the cypinid gallwasp Andricus quercuscalicis (Hymenoptera: Cynipidae). Mol. Ecol., 2, 251-268.

TAYLOR, C. E. AND GORMAN, G. C. 1975. Population genetics of a 'colonising' lizard: natural selection for allozyme morphs in Anolis grahami. Heredity, 35, 241-247.

TEMPLE, s. 1986. Why endemic island birds are so vulnerable to extinction. In: Temple, S. (ed.) Bird Conservation 2, pp. 3-6. International Council for Bird Preservation, US Section: University of Wisconsin Press, Madison, WI.

TSUMURA, Y. AND OHBA, K. 1993. Genetic structure of geographical marginal populations of Cryptomeria japonica. Can. J. Forest Res., 23, 859-863.

VITOUSEK, P. M. 1988. Diversity and biological invasions of oceanic islands. In: Wilson, E. O. and Peters, F. M. (eds) Biodiversity, pp. 181-189. National Academy Press, Washington, DC.

WARNER, R. E. 1968. The role of introduced diseases in the extinction of the endemic Hawaiian avifauna. Condor, 70, 101-120.

WAYNE, R. K., GEORGE, S. B., GILBERT, D., COLLINS, P. W., KOVACH, S. D., GIRMAN, D. AND LEHMAN, N. 1991a. A morphological and genetic study of the island fox, Urocyon littoralis. Evolution, 45, 1849-1868.

WAYNE, R. K., LEHMAN, N., GIRMAN, D., GOGAN, P. J. P., GILBERT, D. A., HANSEN, K. ET AL. 1991b. Conservation genetics of the endangered Isle Royale gray wolf. Consen. Biol., 5, 41-51.

WEBSTER, T. P., SELANDER, R. K. AND YANG, S. Y. 1972. Genetic variability and similarity in the Anolis lizards of Bimini. Evolution, 26, 523-535.

WENDEL, J. F. AND PERCIVAL, A. E. 1990. Molecular diver- 
gence in the Galapagos Islands-Baja California species pair, Gossypium klotzschianum and G. davidsonii (Malvaceae). Pl. Syst. Evol., 171, 99-115.

WENDEL, J. F. AND PERCY, R. G. 1990. Allozymic diversity and introgression in the Galapagos Islands endemic Gossypium darwinii and its relationship to continental G. barbadense. Biochem. Syst. Ecol., 18, 517-528.

WENDEL, J. F., BRUBAKER, C. L. AND PERCIVAL, E. A. 1992.

Genetic diversity in Gossypium hirsutum and the origin of upland cotton. Am. J. Bot., 79, 1291-1310.

wilson, E. O. 1994. Naturalist. Island Press, Shearwater Books, Washington, DC.

woODRUFF, D. S. 1975. Allozyme variation and genic heterozygosity in the Bahaman pulmonate snail Cerion bendalli. Malacolog. Rev., 8, 47-55.

WORLD CONSERVATION MONITORING CENTRE. 1992. Global Biodiversity: Status of the Earth's Living Resources. Chapman and Hall, London.

WRIGHT, s. 1931. Evolution in Mendelian populations. Genetics, 16, 97-159.

ZANETTO, A. AND KREMER, A. 1995. Geographic structure of gene diversity in Quercus petraea (Matt.) Liebl. I. Monolocus patterns of variation. Heredity, 75, 506-517.

ZINK, R. M., LOTT, D. F. AND ANDERSON, D. W. 1987. Genetic variation, population structure, and evolution of California Quail. Condor, 89, 395-405. 